\title{
Risk assessment method for marine net- work security base on fuzzy-gray system theory
}

\author{
Guoliang Zou, Jie Wu, Yueying Jing \\ College of Information Technology, Shanghai Ocean University Shanghai, China
}

\begin{abstract}
This paper constructs a marine network risk assessment model based on fuzzygray system theory, combining fuzzy mathematics with gray system theory. According to some special characters of the Marine Network, we screened the score results and normalized on the index weight with expert scoring. Whereafter, we analyzed the safety index and the object and established gray evaluation matrix based on gray relation analysis theory. Finally we assessed the security of the Marine Network with 2-levels fuzzy comprehensive evaluation method. The method was demonstrated much more scientific, subjective and effective.
\end{abstract}

Key words: Marine Network, FuzzyGray System Method, Risk Assessment

\section{Introduction}

The establishment of the Marine Public Information Platform can effectively promote the development of Marine science and economic. The application of informationization and networkization realized an interchange between each department, information sharing, data integration, and so on. However, it also brings new danger and threat of the information security.

Due to the particularity and sensibility of the marine assets, marine information security affects economic and manage- ment of marine, and the damage and leak of the marine confidential data will even fall the whole country into terrible threats. It is the most urgent and important to effectively put the existing information safety protection technology into the field of the ocean.

\section{Fuzzy-Graysystem Method}

This paper constructs a marine network risk assessment model based on fuzzygray system theory, combining fuzzy mathematics with gray system theory. Diagram of the model is shown in figure 1. It includes five basic-modules and three knowledge bases.

Fig 1: Diagram of the Fuzzy-Gray system method

a) Assets Identification was made to classify the asset category and attribute of Marine Network, referring to the Expert Knowledge Base.

b) Assessment Index Selection was used for select suitable indexes to analyze the network security factors and to acquire a three-layer evaluation system from Index Evaluation Assemble, which based on the modified Delphi method.

c) Index Weight Analysis, based on Expert Knowledge Base, was made to calculate important relative assignment among all the index weight values with the whitenization weight function of gray system theory.

d) Fuzzy Comprehensive Evaluation 
was used to calculate assess weight set and evaluation matrix by fuzzy mathematic to get a comprehensive evaluation result.

e) Risk Value Output was made to be put into the results, which was stored in Network Security Analysis Result Base.

f) Index Evaluation Assemble will provide initial indicators, which also can update or delete the indexes along with the development of the network.

g) Network Security Analysis Result Base will store the process of evaluation, including record of information of the experts who participate in the evaluation, assignment process, the rating of the network system and the security situation analysis, etc. All of these values can update expert knowledge base.

h) Expert Knowledge Base will involve the information of the evaluation objects, such as management system, service, security attributes, etc. Expert Knowledge Base is basis of the comprehensive evaluation, which is also fundamental for the experts to evaluate relative index.

\section{Establishment Of The Index System}

\subsection{Index System Analysis}

Establishment of index system needs to sufficiently understand the each part of the network. In order to understand the network, firstly, the structure and management of the network needs to be analyzed from both aspects of the technology and management. Secondly, the vulnerability of the each part of the network should be analyzed to ascertain existing security measures. Finally, threats should be foreseen, which are likely to cause damage to the network.

\subsection{Assign Index Weight}

It is not easy to quantify the process of assignment of the each index, which was subdivided in the marine network. Therefore, membership functions should be established based on fuzzy math. There are many methods to confirm the functions, such as Probability Statistics, Fuzzy Statistics Testing, Binary Compared Sorting, etc.

Because the evaluation process relies on the experience of experts of marine industry to the great extent, we constituted subjection function, adopting membership values given directly by the experts.

Suppose that we got matrix $\left(W_{h i}\right)_{n \times m}$ from $\mathrm{n}$ experts to assign weights, based on $\mathrm{m}$ indexes. Thus, for the same index $\mathrm{i}$, the similarity coefficient $R_{h g}$ between the expert $\mathrm{h}$ and $\mathrm{g}$ is showed as follows.

$$
R_{h g}=1-\left(\frac{1}{m} \sum_{i=1}^{m}\left(W_{h i}-W_{g i}\right)^{2}\right)^{\frac{1}{2}}
$$

Similarity matrix $\left(R_{h g}\right)_{n \times m}$ came from similarity coefficient $R_{h g}$. The deviation is named with $P_{h}, P_{h}=\sum R_{h g}$. Set a matrix $P=\left(p_{1}, p_{2}, \ldots, p_{n}\right)^{T}$. Suppose that the deviation coefficient is $D_{h}$,

$$
D_{h}=\frac{p_{\max }-p_{h}}{p_{\max }} \times 100 \%
$$

Deviation threshold is $\mathrm{D}_{0}$, if $\mathrm{D}_{\mathrm{h}}>\mathrm{D}_{0}$, thus, expert h's assignment will be eliminated, the other results will be conducted normalization, then we can get the evaluation index weight,

$A=\left\{a_{1}, a_{2}, \ldots, a_{m}\right\}, \sum_{i=1}^{m} a_{i}=1$

Similarly available, we can get each of source of risk $U_{i}$, its weight is $\mathrm{A}_{\mathrm{i}}=\left\{\mathrm{a}_{\mathrm{i} 1}, \mathrm{a}_{\mathrm{i} 2}, \ldots, \mathrm{a}_{\mathrm{in}}\right\}, \quad \sum_{i=1}^{m} a_{i j}=1$ 
After scoring the indexes, the security indexes and objects will be analyzed by gray correlation analysis theory to set a gray evaluation matrix to make fuzzy comprehensive evaluation. Gray classes mean all the possibilities of evaluation objects. Whitenization function can quantitatively describe degrees of belonging to gray classes.

\section{Comprehensive Evaluation}

\subsection{The First Grade Evaluation}

After acquiring Ui evaluation weights set and evaluation matrix, we will calculate value of the comprehensive evaluation.

$$
B_{i}=A_{i} \cdot R_{i}
$$$$
=\left(a_{i 1}, a_{i 2}, \ldots, a_{i m}\right)\left(\begin{array}{cccc}
r_{i 11} & r_{i 12} & \cdots & r_{i 1 k} \\
r_{i 21} & r_{i 22} & \cdots & r_{i 2 k} \\
\vdots & \vdots & \vdots & \vdots \\
r_{i n 1} & r_{i n 2} & \cdots & r_{i n k}
\end{array}\right)
$$

$(i=1,2, \ldots, m)$

\subsection{The Second Grade Evaluation}

The first grade aims at evaluating risk factors of the sub-goal layer $U_{i}$, and the second grade mainly evaluates the risk set of the destination layer $\mathrm{U}$.

$$
B=A \cdot R=\left(a_{1}, a_{2}, \ldots, a_{m}\right)\left(\begin{array}{cccc}
b_{11} & b_{12} & \cdots & b_{1 k} \\
b_{21} & b_{22} & \cdots & b_{2 k} \\
\vdots & \vdots & \vdots & \vdots \\
b_{n 1} & b_{n 2} & \cdots & b_{n k}
\end{array}\right)
$$

\section{Risk Rank}

Risk rank is to visualize the safe condition of the marine network. This paper will be divided into five risk levels, namely very high, high, medium, low and very low, which will show its damage degree with the value of 5,4,3,2,1 respectively.

\section{An Application Example}

The paper will make a risk assessment of marine network in the construction with the application of the fuzzy-gray theory. Because the marine network should be met with above protection level -3 , we will analyze the evaluation indexes and invite five experts to assign relative values according to the Delphi method, to establish the evaluation index system. The index weight is illustrated by Table 1 .

Table 1 Evaluation index weight

\begin{tabular}{|l|l|l|c|}
\hline \multicolumn{1}{|c|}{$\begin{array}{c}\text { Assess } \\
\text { elements }\end{array}$} & weight & \multicolumn{1}{c|}{ factors } & weight \\
\hline \multirow{4}{*}{$\begin{array}{l}\text { Technical } \\
\text { factors }\end{array}$} & \multirow{2}{*}{0.4} & $\begin{array}{l}\text { Physical Envi- } \\
\text { ronment }\end{array}$ & 0.2 \\
\cline { 3 - 4 } & & Network & 0.2 \\
\cline { 3 - 4 } & & Application & 0.1 \\
\cline { 3 - 4 } & Data security & 0.1 \\
\cline { 3 - 4 } Manage- & Host security & 0.4 \\
\hline \multirow{2}{*}{$\begin{array}{l}\text { ment } \\
\text { factors }\end{array}$} & \multirow{2}{*}{0.6} & $\begin{array}{l}\text { System operation } \\
\text { and maintenance }\end{array}$ & 0.3 \\
\cline { 3 - 4 } & $\begin{array}{l}\text { Network system } \\
\text { construction }\end{array}$ & 0.3 \\
\cline { 3 - 4 } & & Safety institution & 0.4 \\
\hline
\end{tabular}

Assume the assess set $V=\{1,2,3,4,5\}$, which means that the safety risk level of the evaluation indexes is marked for high-risk, danger, general, low-risk, and safety. The sample matrix is as following.

Table 2 Sample matrix

\begin{tabular}{|c|c|c|c|c|c|}
\hline $\mathrm{U}_{1}$ & $\mathrm{U}_{11}$ & $\mathrm{U}_{12}$ & $\mathrm{U}_{13}$ & $\mathrm{U}_{14}$ & $\mathrm{U}_{15}$ \\
\hline $\mathrm{p}_{1}$ & 3 & 4 & 2 & 1 & 2 \\
\hline $\mathrm{P}_{2}$ & 2 & 3 & 1 & 3 & 2 \\
\hline $\mathrm{P}_{3}$ & 3 & 2 & 3 & 3 & 2 \\
\hline $\mathrm{P}_{4}$ & 4 & 3 & 2 & 2 & 3 \\
\hline $\mathrm{P}_{5}$ & 2 & 3 & 1 & 4 & 2 \\
\hline
\end{tabular}

\begin{tabular}{|c|c|c|c|}
\hline $\mathrm{U}_{2}$ & $U_{21}$ & $\mathrm{U}_{22}$ & $\mathrm{U}_{23}$ \\
\hline $\mathrm{p}_{1}$ & 3 & 1 & 2 \\
\hline $\mathrm{P}_{2}$ & 4 & 3 & 2 \\
\hline $\mathrm{P}_{3}$ & 3 & 2 & 3 \\
\hline $\mathrm{P}_{4}$ & 4 & 3 & 2 \\
\hline $\mathrm{P}_{5}$ & 2 & 3 & 1 \\
\hline
\end{tabular}


The following whitenization weight functions can be derived by the gray theory.

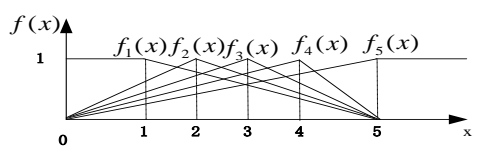

Fig 2 Whitenization weight function

Each whitenization weight function of the gray class $h$ can be expressed as following,

$$
\begin{aligned}
& f_{1}(x)=\left\{\begin{array}{l}
1, x \in[0,1) \\
(5-x) / 4, x \in[1,5]
\end{array}\right. \\
& f_{2}(x)=\left\{\begin{array}{l}
x / 2, x \in[0,2) \\
(5-x) / 3, x \in[2,5]
\end{array}\right. \\
& f_{3}(x)=\left\{\begin{array}{l}
x / 3, x \in[0,3) \\
(5-x) / 2, x \in[3,5]
\end{array}\right. \\
& f_{4}(x)=\left\{\begin{array}{l}
x / 4, x \in[0,4) \\
5-x, x \in[4,5]
\end{array}\right. \\
& f_{5}(x)=\left\{\begin{array}{l}
x / 5, x \in[1,5) \\
1, x \geq 5
\end{array}\right.
\end{aligned}
$$

Thus, $\mathrm{U}_{11}$ 's gray evaluation coefficient $\mathrm{r} 11$ of the first gray class can be acquired as following.

$$
r_{11}=\frac{\sum_{p=1}^{5} f_{h}\left(a_{i j p}\right)}{\sum_{h=1}^{5} \sum_{p=1}^{5} f_{h}\left(a_{i j p}\right)}
$$

From the calculation, $r_{11}=(0.166$, $0.222, \quad 0.232, \quad 0.211, \quad 0.169)$.Similarly available, we can get the other evaluation coefficients, based on the above values, we can establish evaluation matrixes $R_{i}$.

$$
\begin{aligned}
R_{1} & =\left[\begin{array}{lllll}
0.166 & 0.222 & 0.232 & 0.211 & 0.168 \\
0.154 & 0.205 & 0.226 & 0.231 & 0.185 \\
0.272 & 0.249 & 0.204 & 0.153 & 0.122 \\
0.196 & 0.207 & 0.230 & 0.212 & 0.157 \\
0.209 & 0.278 & 0.218 & 0.164 & 0.131
\end{array}\right] \\
R_{2} & =\left[\begin{array}{lllll}
0.140 & 0.186 & 0.228 & 0.248 & 0.199 \\
0.195 & 0.206 & 0.285 & 0.177 & 0.141 \\
0.238 & 0.265 & 0.212 & 0.159 & 0.141
\end{array}\right]
\end{aligned}
$$

By the first grade evaluation, we can get

$$
B_{i}=A_{i} \cdot R_{i}
$$

$\mathrm{B}_{1}=(0.194,0.242,0.222,0.191,0.151)$,

$\mathrm{B}_{2}=(0.196,0.224,0.239,0.191,0.158)$ 。

Then by the second grade evaluation, we can get $B=A \cdot R$,

$$
R=\left(\begin{array}{lllll}
0.194 & 0.242 & 0.222 & 0.191 & 0.151 \\
0.196 & 0.224 & 0.239 & 0.191 & 0.158
\end{array}\right)
$$

Based on the values, we can get the available result as following.

$\mathrm{B}=(0.1952,0.2312,0.2322,0.191$, $0.1552)$

According to percentage conversion,

$\mathrm{C}=(20,40,60,80,100)^{\mathrm{T}}, S=B \cdot C$,

Finally we can get the value of the comprehensive evaluation of the whole network, which were 57.84. Its risk level is medium.

\section{SUMMARIZE}

In the process of the information safety assessment, because of risk factors with strong fuzziness and uncertainty, its valid value can't be effectively accumulated simply. This paper tried to establish the fuzzy-gray system model to design the process of risk assessment of marine network, providing a reference for producing a risk assessment system in the future. Meanwhile, we calculated the index weight assignment by fuzzy-gray, which made the assignment more objective and scientific, with possible human errors reduced and the evaluation results corresponding to the facts.

\section{References}

[1] LiuJian-hua, Hou Hong-xia, Wu Hao. Method to Determine Weight in Se- 
curity Evaluation of Telecommunication Data Network, 2010.

[2] Wang Hongli, Feng Yuqiang, Ablation Model Based on the Improved Gray Cloud and Its Applicant in the Gray Decision, 2006,23 ( 6 ) : 740-745.

[3] ISO/IEC 15408-1(1999-12), Information Technology-Security Tech-
niques-Common Criteria for IT Security Evaluation (CCITSE), 1999.

[4] BS7799-2:1999, Information Security Management. Specification for Information Security Management Systems, British Standards Institute, 1999.

[5] Information Safe Risk Assessment Report. The state council information office , 2003 